\title{
The Nucleus Accumbens Core is Necessary to Scale Fear to Degree of Threat
}

\author{
${ }^{\circledR}$ Madelyn H. Ray, Alyssa N. Russ, ${ }^{\circledR}$ Rachel A. Walker, and ${ }^{\circledR}$ Michael A. McDannald \\ Department of Psychology and Neuroscience, Boston College, Chestnut Hill, MA 02467
}

Fear is adaptive when the level of the response rapidly scales to degree of threat. Using a discrimination procedure consisting of danger, uncertainty, and safety cues, we have found rapid fear scaling (within $2 \mathrm{~s}$ of cue presentation) in male rats. Here, we examined a possible role for the nucleus accumbens core (NAcc) in the acquisition and expression of fear scaling. In experiment 1, male Long-Evans rats received bilateral sham or neurotoxic NAcc lesions, recovered, and underwent fear discrimination. NAcc-lesioned rats were generally impaired in scaling fear to degree of threat, and specifically impaired in rapid uncertainty-safety discrimination. In experiment 2, male Long-Evans rats received NAcc transduction with halorhodopsin (Halo) or a control fluorophore. After fear scaling was established, the NAcc was illuminated during cue or control periods. NAcc-Halo rats receiving cue illumination were specifically impaired in rapid uncertainty-safety discrimination. The results reveal a general role for the NAcc in scaling fear to degree of threat, and a specific role in rapid discrimination of uncertain threat and safety.

Key words: fear; nucleus accumbens; optogenetics; threat

\section{Significance Statement}

Rapidly discriminating cues for threat and safety is essential for survival and impaired threat-safety discrimination is a hallmark of stress and anxiety disorders. In two experiments, we induced nucleus accumbens core (NAcc) dysfunction in rats receiving fear discrimination consisting of cues for danger, uncertainty, and safety. Permanent NAcc dysfunction, via neurotoxic lesion, generally disrupted the ability to scale fear to degree of threat, and specifically impaired one component of scaling: rapid discrimination of uncertain threat and safety. Reversible NAcc dysfunction, via optogenetic inhibition, specifically impaired rapid discrimination of uncertain threat and safety. The results reveal that the NAcc is essential to scale fear to degree of threat, and is a plausible source of dysfunction in stress and anxiety disorders.

\section{Introduction}

The ability to discriminate danger from safety is critical to survival. Individuals with stress and anxiety disorders are impaired in discrimination, showing excessive fear-related responses to safety (Jovanovic et al., 2010, 2012; Lissek et al., 2014; Duits et al., 2015). Danger and safety represent extremes of a threat continuum, with most real-world threats involving uncertainty. Ideally, one's level of fear should scale to degree of threat. A scaled fear response would be most adaptive if it was rapidly organized following encounter with a potential threat.

Received Feb. 6, 2020; revised Apr. 23, 2020; accepted Apr. 27, 2020.

Author contributions: M.H.R. and M.A.M. designed research; M.H.R., A.N.R., and R.A.W. performed research; M.H.R. and M.A.M. analyzed data; M.H.R., R.A.W., and M.A.M. wrote the paper.

This work was supported by National Institutes of Health Grant DA034010 (to M.A.M.). We thank Alexa LaBanca and Andrew Thomson for technical support. We also thank Bret Judson and the Boston College Imaging Core for infrastructure and support.

The authors declare no competing financial interests.

Correspondence should be addressed to Michael A. McDannald at michael.mcdannald@bc.edu or Madelyn H. Ray at madelyn.ray@bc.edu.

https://doi.org/10.1523/JNEUROSCI.0299-20.2020

Copyright $(2020$ the authors
Drawing from learning theory (Rescorla, 1968), our laboratory devised a discrimination procedure in which distinct auditory cues predict unique foot shock probabilities: danger $(p=1.00)$, uncertainty $(p=0.25)$, and safety $(p=0.00$; Berg et al., 2014). Using this procedure, we have found that fear level scales to shock probability within $2 \mathrm{~s}$ of cue presentation (DiLeo et al., 2016). Present work in our laboratory seeks to identify brain regions necessary for fear scaling and its rapid emergence. Candidate regions should signal valence and receive amygdalar input (Quirk et al., 1995; Goosens and Maren, 2001; Koo et al., 2004; McDannald and Galarce, 2011). We identified the nucleus accumbens core (NAcc) as a likely candidate, based on its ability to rapidly signal relative reward value (Cromwell and Schultz, 2003; Setlow et al., 2003; Ambroggi et al., 2011; McGinty et al., 2013; Saddoris and Carelli, 2014; Sugam et al., 2014; Ottenheimer et al., 2018), as well as its anatomic connectivity with the amygdala (Kita and Kitai, 1990; Petrovich et al., 1996; Wright and Groenewegen, 1996). Even more, the NAcc is implicated in a variety of fear-related processes (Haralambous and Westbrook, 1999; Thomas et al., 2002; Schwienbacher et al., 2004; Iordanova et al., 2006b; Fadok et al., 2010; Badrinarayan et al., 2012; Oleson et al., 2012; Li and McNally, 2015; Correia et al., 2016). 
In two experiments, we examined roles for the NAcc in fear scaling. In experiment 1 , we permanently ablated NAcc neurons via neurotoxic lesion. Following recovery, rats received fear discrimination consisting of danger, uncertainty, and safety cues. Fear was measured with suppression of rewarded nose poking (Estes and Skinner, 1941; Bouton and Bolles, 1980). Examining nose poke suppression over the entire 10-s cue permitted analysis of overall fear scaling. To scrutinize the temporal emergence of scaling, we divided the 10 -s cues into five, 2 -s intervals. Focusing on suppression during the first 2-s cue interval permitted analysis of rapid fear scaling. In experiment 2, we transducted NAcc neurons with halorhodopsin (Halo) or a control fluorophore, and implanted ferrules bilaterally above the transducted NAcc neurons. Following recovery, rats received discrimination until fear scaling was stable. Over the next eight sessions, the NAcc was green-light illuminated during cue presentation or a control period, optogenetically inhibiting NAcc activity in Halo rats. The two experiments allowed us to uncover roles for the NAcc in the acquisition and expression of fear scaling.

\section{Materials and Methods}

\section{Experiment 1}

Subjects

Subjects were forty-five male Long-Evans rats weighing 275-300 g on arrival (Charles River Laboratories; RGD catalog \# 2308852, RRID: RGD_2308852). Rats were individually housed and maintained on a 12/12 h light/dark cycle (lights off at 6 P.M.) with water ad libitum. Procedures adhered to the NIH Guide for the Care and Use of Laboratory Animals and were approved by the Boston College Institutional Animal Care and Use Committee.

\section{Behavioral apparatus}

Eight sound-attenuated enclosures each housed a behavior chamber with aluminum front and back walls, clear acrylic sides and top, and a metal grid floor. Grid floors were electrically connected to a shock generator. A single external food cup and central nose poke opening equipped with infrared photocells were present on one wall. Auditory stimuli were presented through two speakers mounted on the ceiling of each behavior chamber.

\section{Surgical procedures}

Stereotaxic surgery was performed under isoflurane anesthesia (2-5\%) using aseptic technique. Twenty-four rats received bilateral infusions of $N$-Methyl-D-aspartic acid $(15 \mu \mathrm{g} / \mu \mathrm{l}$ in Dulbecco's PBS) aimed at the NAcc $(0.40 \mu \mathrm{l},+1.90 \mathrm{AP}, \pm 1.80 \mathrm{ML},-6.60 \mathrm{DV}$ from skull). Infusions were delivered via $2-\mu l$ syringe (Hamilton, Neuros) controlled by a microsyringe pump (World Precision Instruments, UMP3-2). Infusion rate was $\sim 0.11 \mu \mathrm{l} / \mathrm{min}$. Thirty seconds after the completion of each infusion, the syringe was raised $0.1 \mathrm{~mm}$, then left in place for $5 \mathrm{~min}$ to encourage delivery to the target site. The remaining twenty-one rats received identical surgical treatment without infusions. Rats received carprofen $(5 \mathrm{mg} / \mathrm{kg})$ for postoperative analgesia.

\section{Nose poke acquisition}

Following recovery from surgery, rats were food restricted to $85 \%$ of their initial free feeding body weight, then fed $(2-20 \mathrm{~g} / \mathrm{d})$ to increase their target body weight by $1 \mathrm{~g} / \mathrm{d}$ for the remainder of testing. Rats were shaped to nose poke for pellet (BioServ F0021, protein/fat/carbohydrate blend) delivery using a fixed ratio 1 schedule: one nose poke yielded one pellet. Shaping sessions lasted $30 \mathrm{~min}$ or $\sim 50$ nose pokes. Over the next three, 60 -min sessions, rats were placed on variable interval (VI) schedules in which nose pokes were reinforced on average every $30 \mathrm{~s}$ (session 1) or $60 \mathrm{~s}$ (sessions 2 and 3). For the remainder of testing, nose pokes were reinforced on a VI-60 schedule independent of all Pavlovian contingencies.

\section{Preexposure}

In two separate sessions, each rat was preexposed to the three cues to be used in Pavlovian fear discrimination. Cues were auditory stimuli, 10-s in duration and consisted of repeating motifs of a broadband click, phaser, or trumpet. Stimuli can be heard or downloaded at http:// mcdannaldlab.org/resources/ardbark. Previous studies have found these stimuli to be equally salient, yet highly discriminable (Berg et al., 2014; Wright et al., 2015; DiLeo et al., 2016; Ray et al., 2018). The 42-min preexposure sessions consisted of four presentations of each cue (12 total presentations) with a mean intertrial interval (ITI) of $3.5 \mathrm{~min}$. The order of trial type presentation was randomly determined by the behavioral program and differed for each rat during each session throughout behavioral testing.

For all sessions, fear to each auditory cue was measured using a suppression ratio based on nose poke rates during the 20-s baseline period immediately preceding the 10 -s cue period: suppression ratio $=$ (baseline nose poke rate - cue nose poke rate)/(baseline nose poke rate + cue nose poke rate). A ratio of 1 indicated complete suppression of nose poking during the cue and a high level of fear; 0 , no suppression and no fear. Intermediate suppression ratios reflected intermediate fear levels. The same suppression ratio formula was used to calculate fear in 2-s cue intervals.

\section{Fear discrimination}

Each rat received 16, 54-min Pavlovian fear discrimination sessions. Sessions began with a $\sim 5$ min warm-up period during which no cues or shock were presented. The three cues were associated with a unique foot shock (0.5 mA, 0.5-s) probability: danger (1.00), uncertainty (0.25), and safety (0.00). Foot shock was administered 1-s following cue offset. A single session consisted of four danger, six uncertainty omission, two uncertainty shock, and four safety trials. Auditory stimulus identity was counterbalanced across rats. Mean ITI was $3.5 \mathrm{~min}$.

\section{Histology}

Upon the conclusion of behavior, rats were anesthetized with an overdose of isoflurane and perfused intracardially with $0.9 \%$ biological saline. Brains were extracted and stored in $4 \%(\mathrm{v} / \mathrm{v})$ formalin and $10 \%(\mathrm{w} / \mathrm{v}) \mathrm{su}-$ crose. Forty-micrometer sections were collected on a sliding microtome. Tissue was then washed with PBS, incubated in NeuroTrace (Thermo Fisher Scientific, N21479) at a 1:200 concentration, washed again, mounted, dried, and coverslipped with Vectashield Hardset mounting media (Vector Labs, H-1400). Slides were imaged within three weeks of processing.

\section{Experiment 2}

Subjects

Subjects were 25 male Long-Evans rats weighing 275-300 g on arrival (Charles River Laboratories; RGD catalog \#2308852, RRID:RGD_ 2308852). Rats were individually housed and maintained on a $12 / 12 \mathrm{~h}$ light/dark cycle (lights off at 6 P.M.) with water ad libitum. Procedures adhered to the NIH Guide for the Care and Use of Laboratory Animals and were approved by the Boston College Institutional Animal Care and Use Committee.

\section{Behavioral apparatus}

Behavior chambers were identical to experiment 1 , only now each chamber was equipped with a green laser $(532 \mathrm{~nm}$, max $500 \mathrm{~mW}$; Shanghai Laser \& Optics Century Co, Ltd.) to illuminate the NAcc. The laser was controlled by digital pulses from the Med Associates Computer. The complete green light pathway was: laser $\rightarrow$ patch cord $\rightarrow 1 \times 2$ fiber optic rotatory joint (Doric) $\rightarrow$ shielded, bilateral fiber-optic cable $\rightarrow$ implanted fiber-optic ferrules. A ceramic sleeve maintained tight contact between the cable and ferrule. The cable-ferrule junction was surrounded by black shrink wrap to reduce light emission into the behavioral chamber. A PM160 light meter (Thorlabs) was used to measure and calibrate light output before each illumination session. 


\section{Optogenetic materials}

Optical ferrules were constructed using 2.5-mm ceramic zirconia ferrules (Precision Fiber Products). Behavior cables were custom made for light delivery (Multimode Fiber, 0.22 NA, High-OH, Ø200 $\mu \mathrm{m}$ Core). Protocols can be downloaded at http://mcdannaldlab.org/resources/ optogenetics.

\section{Surgical procedures}

Stereotaxic surgery was performed under isoflurane anesthesia $(2-5 \%)$ using aseptic technique. Thirteen rats received bilateral infusions of AAV-hSyn-eNpHR3.0-EYFP (halorhodopsin) aimed at the NAcc $\left(0.50 \mu \mathrm{l},+1.90 \mathrm{AP}, \pm 1.80 \mathrm{ML},-6.60 \mathrm{DV}\right.$ at a $0^{\circ}$ angle $)$ and bilateral optical ferrules ( $+1.70 \mathrm{AP}, \pm 2.80 \mathrm{ML},-6.00 \mathrm{DV}$ at a $10^{\circ}$ angle). Infusions were delivered via $2-\mu \mathrm{l}$ syringe (Hamilton, Neuros) controlled by a microsyringe pump (World Precision Instruments, UMP3-2). Infusion rate was $\sim 0.11 \mu \mathrm{l} / \mathrm{min}$. The syringe was raised $0.1 \mathrm{~mm}$ after each infusion, then left in place for $5 \mathrm{~min}$ to encourage delivery to the target site. The remaining 12 rats received identical surgical treatment but were infused with a control fluorophore (AAV-hSyn-EYFP). Implants were secured with dental cement surrounded by a modified, $50-\mathrm{ml}$ centrifuge tube. After surgery, rats received two weeks of undisturbed recovery with prophylactic antibiotic treatment (cephalexin; Henry Schein Medical 049167) before beginning nose poke acquisition. All rats received carprofen $(5 \mathrm{mg} / \mathrm{kg})$ for postoperative analgesia.

\section{Preillumination training and cable habituation}

Nose poke acquisition, preexposure, and initial fear discrimination (10 sessions) were identical to experiment 1 . We increased the delay between cue offset and shock onset to $2 \mathrm{~s}$ to ensure that neural activity would not be inhibited during shock delivery. Cable habituation was provided in two consecutive sessions by plugging rats into optogenetic cables and administering fear discrimination without illumination. In total, rats received twelve fear discrimination sessions before receiving light illumination.

\section{NAcc illumination}

Rats received eight sessions of fear discrimination plus NAcc illumination. The NAcc was illuminated via bilateral delivery of $12.5 \mathrm{~mW}$ of 532-nm "green" light: DPSS laser $\rightarrow$ patch cord $\rightarrow$ commutator $\rightarrow$ optogenetic cables $\rightarrow$ implanted ferrules. There were two types of illumination sessions: cue and ITI. For cue sessions, light illumination began $0.5 \mathrm{~s}$ before cue onset and ended $0.5 \mathrm{~s}$ following cue offset, resulting in a total illumination time of $11 \mathrm{~s}$. Light illumination was given on all trial types (danger, uncertainty, and safety) for a total of 16 illumination events per session. For ITI sessions, illumination occurred during the ITIs between cue presentations. Illumination was roughly equidistant from previous cue offset and subsequent cue onset ( $\sim 90 \mathrm{~s}$ from each). Sixteen ITI illumination events were administered, each lasting $11 \mathrm{~s}$, equating total illumination time for cue and ITI sessions. The within-subjects design meant that each rat received four cue illumination sessions and four ITI illumination sessions. Illumination was given in two-session blocks, with roughly half of the subjects starting with ITI illumination.

\section{Histology}

After behavioral testing ended, rats were anesthetized with an overdose of isoflurane and perfused intracardially with $0.9 \%$ biological saline and $4 \%$ paraformaldehyde in a $0.2 \mathrm{M}$ potassium PBS. Brains were extracted and stored in $4 \%(\mathrm{v} / \mathrm{v})$ formalin and $10 \%(\mathrm{w} / \mathrm{v})$ sucrose. Forty-micrometer sections were collected on a sliding microtome. Tissue was rinsed, incubated in NeuroTrace, rinsed again, mounted, dried, and coverslipped with Vectashield Hardset (Vector Labs, H-1400). Slides were imaged within three weeks of processing.

\section{Statistical analysis}

Behavioral data were acquired using Med Associates Med-PC IV software (MED PC, RRID: SCR_012156). Raw data were processed in MATLAB (RRID: SCR_001622) to extract time stamps for nose poke and cue onset. Suppression ratios were calculated as: (baseline poke rate - cue poke rate)/(baseline poke rake + cue poke rate) and were analyzed with repeated measures ANOVA in SPSS (RRID:SCR_002865). Repeated measures ANOVA was performed with factors of group, cue, and time (experiment 1) and group, cue, time, and illumination (experiment 2). Partial $\eta^{2}\left(\eta_{\mathrm{p}}^{2}\right)$ and observed power (op) are reported for ANOVA results for indicators of effect size. For all analyses, $p<0.05$ (or an appropriate Bonferroni correction) was considered significant.

\section{Results}

\section{Experiment 1}

Histologic results

Rats received bilateral sham or neurotoxic NAcc lesions. Neurotoxic damage (cell loss and gliosis) was quantified. Twenty-four NAcc rats showed damage primarily in the NAcc ( $>85 \%)$ with minor damage ( $\sim 10 \%$ or less) in the neighboring accumbens shell. Shams showed no evidence of neurotoxic damage. Representative sham (Fig. 1A, left), and NAcc lesion (Fig. $1 A$, right) sections are shown. Each subject's lesion was drawn, made transparent, and stacked (Fig. $1 B$ ). Darker areas indicate regions of greater overlap and more consistent damage. Rats fully recovered from surgery before receiving fear discrimination (Fig. 1C).

\section{Baseline nose poking}

NAcc lesions altered the progression of nose poking over discrimination sessions, but did not grossly reduce nose poke rates (Fig. 1D). ANOVA for baseline nose poke rate with session (16) and group (sham vs NAcc) as factors found a main effect of session $\left(F_{(15,645)}=47.14, p=3.77 \times 10^{-93}, \eta_{\mathrm{p}}^{2}=0.52, \mathrm{op}=1.00\right)$, a session $\times$ group interaction $\left(F_{(15,645)}=2.10, p=0.008, \eta_{\mathrm{p}}^{2}=0.05\right.$, op $=0.97)$ but no main effect of group $\left(F_{(1,43)}=0.16, p=0.69\right.$, $\eta_{\mathrm{p}}{ }^{2}=0.004$, op $\left.=0.07\right)$. Dividing the 16 sessions into two, eightsession blocks; ANOVA found a block $\times$ group interaction $\left(F_{(1,43)}=4.81, p=0.034, \eta_{\mathrm{p}}^{2}=0.10\right.$, op $\left.=0.57\right)$. While sham $\left(t_{(20)}=7.69, p=2.13 \times 10^{-7}\right)$ and NAcc rats $\left(t_{(23)}=5.63\right.$, $p=1.00 \times 10^{-5}$ ) both increased poking from the first to second half of discrimination, sham rats showed greater increases $\left(t_{(43)}=\right.$ 2.20, $p=0.034$; Fig. $1 E$ ). Mean \pm SEM baseline nose pokes rates for sessions 1-8: sham (28.44 \pm 2.96$)$ and NAcc (28.83 \pm 1.97$)$; sessions 9-16: sham $(38.80 \pm 3.62)$ and NAcc $(35.33 \pm 2.46)$.

\section{Fear scaling}

Sham rats acquired appropriate scaling of the fear response over the 16 sessions (Fig. $2 A$, left). Suppression ratios for the entire 10 -s cue were low in preexposure and initially increased to all cues. As discrimination proceeded, the suppression ratio for each cue diverged: high to danger, intermediate to uncertainty, and low to safety. NAcc rats showed a similar progression, but poorer overall scaling (Fig. $2 \mathrm{~A}$, right). In support of the general emergence of scaling, ANOVA [between factor: group (sham vs NAcc); within factors: session (16) and cue (danger, uncertainty, and safety)] revealed a main effect of cue $\left(F_{(2,86)}=115.51, p=\right.$ $\left.4.34 \times 10^{-25}, \eta_{\mathrm{p}}^{2}=0.73, \mathrm{op}=1.00\right)$ and a cue $\times$ session interaction $\left(F_{(30,1290)}=14.05, p=6.30 \times 10^{-60}, \eta_{\mathrm{p}}^{2}=0.25\right.$, op $\left.=1.00\right)$. Revealing impaired scaling in NAcc rats, ANOVA found a cue $\times$ group interaction $\left(F_{(2,86)}=5.76, p=0.004, \eta_{\mathrm{p}}^{2}=0.12\right.$, op $\left.=0.86\right)$. The cue $\times$ group interaction was observed when only the last six sessions were analyzed $\left(F_{(2,86)}=4.50, p=0.014, \eta_{\mathrm{p}}^{2}=0.10\right.$, op $=0.76)$, sessions by which scaling patterns were stable.

To further reveal the deficit in NAcc rats, we focused on suppression ratios during the final six sessions. Difference scores were calculated for the two components of scaling: (danger uncertainty) and (uncertainty - safety). Sham (Fig. 2B, left) and $\mathrm{NAcc}$ rats (Fig. 2B, right) discriminated each cue pair. One- 

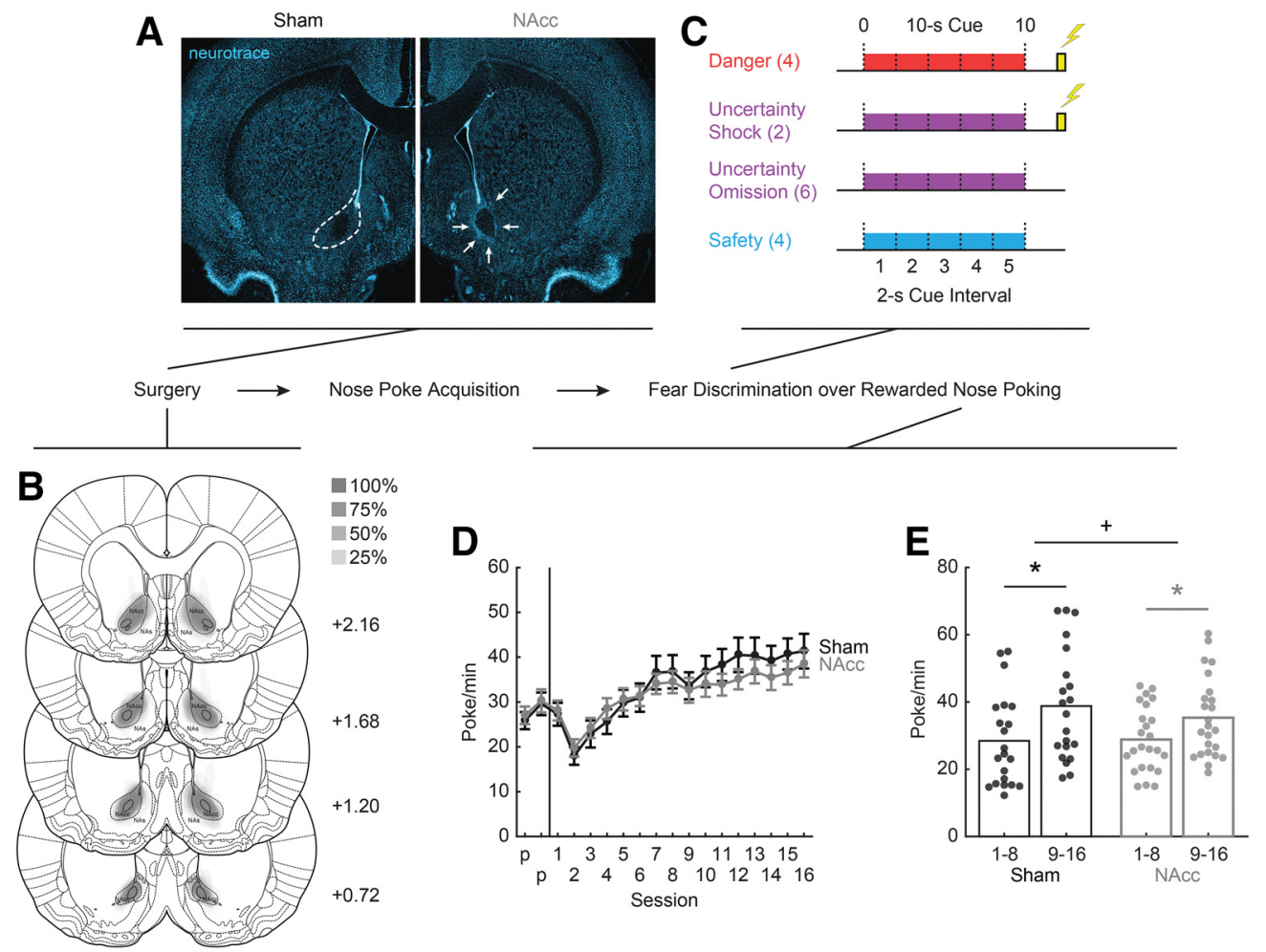

Figure 1. NAcc lesion experimental outline. $A$, Representative sham with NAcc intact (left) and lesion with NAcc damage (right) is shown. Dotted lines (left) show approximate NAcc location. Arrows (right) indicate gliosis and damage restricted to the NAcc. $\boldsymbol{B}$, The extent of neurotoxic NAcc lesions across four coronal planes is shown, and the anterior distance from bregma (millimeters) is indicated. C, Pavlovian fear discrimination consisted of three, 10-s cues predicting unique foot shock probabilities: danger $(p=1.00)$, red; uncertainty $(p=0.25)$, purple; and safety $(p=0.00)$, blue. Cues were divided into five, 2 -s intervals (dotted lines) for rapid analyses. $\boldsymbol{D}$, Mean \pm SEM baseline nose poke rates for the 16 fear discrimination sessions are shown for sham (black) and NAcc (gray) rats. $E$, Mean baseline nose poke rates for sessions 1-8 and 9-16 for sham and NAcc rats. Data points show individual poke rates; ${ }^{*}$ independent samples $t$ test, $p<0.025,{ }^{+}$block $\times$group interaction $p<0.05$. NAs, nucleus accumbens shell.

sample $t$ tests found that difference scores exceeded zero for each comparison: sham, danger versus uncertainty $\left(t_{(20)}=10.25, p=\right.$ $\left.2.07 \times 10^{-9}\right)$, uncertainty versus safety $\left(t_{(20)}=6.11, p=4.17 \times\right.$ $\left.10^{-8}\right)$; NAcc, danger versus uncertainty $\left(t_{(23)}=8.01, p=0.001\right)$, uncertainty versus safety $\left(t_{(23)}=3.65, p=0.002\right)$. However, difference scores were reduced across both components in NAcc rats. ANOVA [between factor: group (sham vs NAcc); within factor: discrimination (danger - uncertainty) and (uncertainty safety)] revealed a main effect of group $\left(F_{(1,43)}=5.68, p=0.022\right.$, $\eta_{\mathrm{p}}^{2}=0.12$, op $\left.=0.64\right)$. These results reveal a general role for the NAcc in fear scaling.

\section{Rapid fear scaling}

We were interested in revealing a possible role for the NAcc in the rapid emergence of fear scaling. To do this, we examined mean suppression ratios during the last six sessions. Each cue was divided into five, 2-s intervals and suppression ratios were calculated for each cue/interval. Sham rats showed scaling of the fear response in the first 2-s cue interval and in all subsequent intervals (Fig. 2C, left). Scaling was reduced across all intervals in NAcc rats (Fig. 2C, right). ANOVA [between factor: group (sham vs NAcc); within factors: interval (five, 2-s cue intervals) and cue (danger, uncertainty, and safety)] found a group $\times$ cue interaction $\left(F_{(2,86)}=3.88, p=0.024, \quad \eta_{\mathrm{p}}^{2}=0.08\right.$, op=0.69). Supporting a specific role for the NAcc in rapid fear scaling, NAcc rats showed impaired scaling even when only the first 2-s cue interval was analyzed (cue $\times$ group interaction; $F_{(2,86)}=5.08$, $\left.p=0.0008, \eta_{\mathrm{p}}^{2}=0.11, \mathrm{op}=0.81\right)$. No cue $\times$ group interaction was observed when the last 2 -s cue interval was analyzed $\left(F_{(2,86)}=1.90\right.$, $p=0.16, \eta_{\mathrm{p}}^{2}=0.04, \mathrm{op}=0.39$ ).
To specify the nature of the deficit in NAcc rats, we reduced scaling into its component parts: (danger - uncertainty) and (uncertainty - safety). We calculated difference scores for the first and last 2-s cue intervals. Sham rats showed positive difference scores for each cue pair at each interval (Fig. 2D). Difference scores exceeded zero, as revealed by one-sample $t$ tests: first 2-s cue interval: danger versus uncertainty $\left(t_{(20)}=\right.$ $\left.10.95, p=6.7 \times 10^{-4}\right)$, uncertainty versus safety $\left(t_{(20)}=3.55\right.$, $p=0.002)$; last 2 -s cue interval: danger versus uncertainty $\left(t_{(23)}=\right.$ 4.60, $\left.p=1.76 \times 10^{-4}\right)$, uncertainty versus safety $\left(t_{(23)}=5.73\right.$, $p=1.30 \times 10^{-5}$ ) for shams. NAcc rats were generally impaired in rapid scaling. ANOVA for the first 2-s cue interval differences revealed a main effect of group $\left(F_{(1,43)}=6.50, p=0.014, \eta_{\mathrm{p}}^{2}=\right.$ 0.01 , op $=0.70$ ), while ANOVA for the last 2-s cue interval difference scores found no main effect $\left(F_{(1,43)}=2.49, p=0.12, \eta_{\mathrm{p}}^{2}=\right.$ 0.05 , op $=0.34$ ). Difference scores also suggest that NAcc rats were more specifically impaired in rapid uncertainty-safety discrimination (Fig. 2D). One-sample tests found that only the NAcc uncertainty-safety difference score from the first 2-s cue interval failed to differ from zero: first interval: danger versus uncertainty $\left(t_{(23)}=4.20, p=3.38 \times 10^{-4}\right)$, uncertainty versus safety $\left(t_{(23)}=1.31, p=0.20\right)$; last interval: danger versus uncertainty $\left(t_{(20)}=5.22, p=2.70 \times 10^{-5}\right)$, uncertainty versus safety $\left(t_{(20)}=4.19, p=3.53 \times 10^{-4}\right)$. All significant, one-sample $t$ tests survive Bonferroni correction $(0.05 / 8, p<0.00625)$.

\section{Experiment 2}

Experiment 1 results reveal a general role for the NAcc in the acquisition of fear scaling and rapid uncertainty-safety 


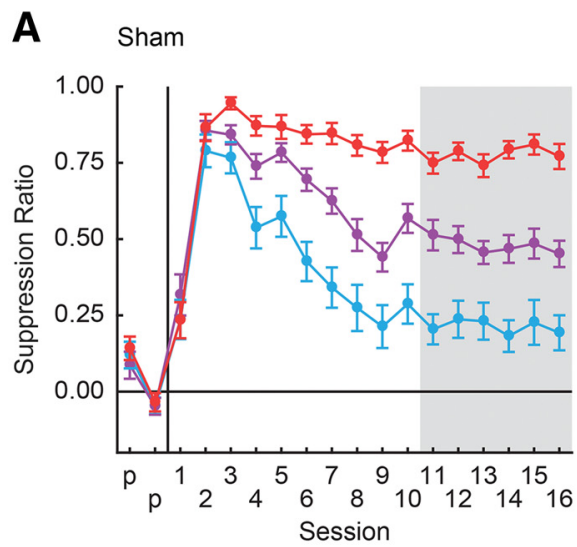

NAcC

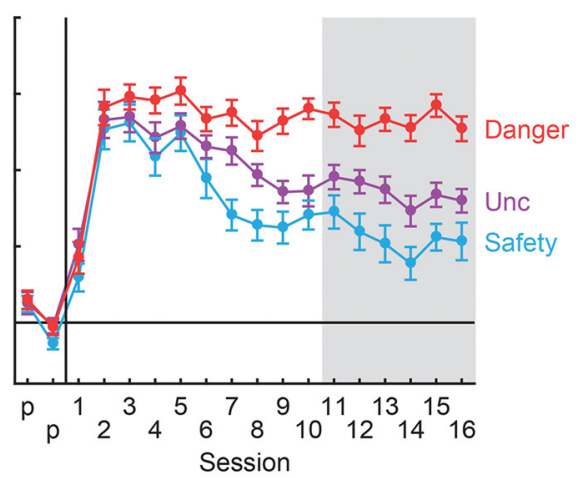

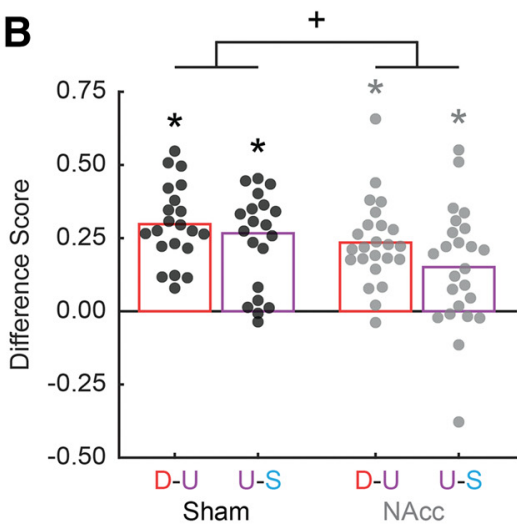
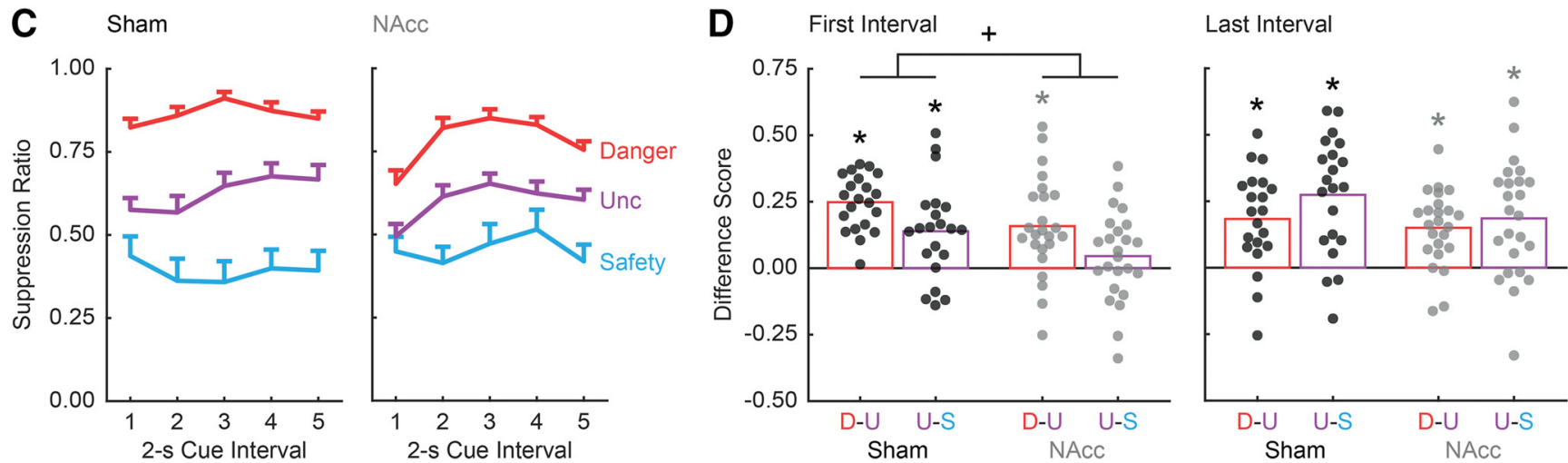

Figure 2. NAcc lesions and fear scaling. $\boldsymbol{A}$, Mean \pm SEM suppression ratio for danger (red), uncertainty (purple), and safety (blue) are shown for sham (left) and NAcc (right) rats. The vertical lines separate the two preexposure and 16 fear discrimination sessions. The last six discrimination sessions are shaded. $\boldsymbol{B}$, Mean difference score for danger versus uncertainty (D-U, red bar) and uncertainty versus safety (U-S, purple bar) across the entire 10-s cue is shown for sham (left) and NAcc (right) rats. Data points show individual difference scores; ${ }^{*}$ one-sample $t$ test compared with zero, $p<0.0125 ;{ }^{+}$main effect of group, $p<0.05$. C, Mean \pm SEM suppression ratios for the five, 2-s cue intervals are shown for sham (left) and NAcc (right) rats. Cue color scheme maintained from $A$. D, Mean difference score for danger versus uncertainty (D-U, red bar) and uncertainty versus safety (U-S, purple bar) is shown for the first 2-s cue interval (left) and last 2-s cue interval (right) for sham and NAcc rats. Data points show individual difference scores; ${ }^{*}$ one-sample $t$ test compared with zero, $p<0.00625 ;{ }^{+}$main effect of group, $p<0.05$.

discrimination. However, neurotoxic lesions altered baseline nose poking and permanently ablated NAcc neurons. To determine a specific role for NAcc cue activity in the expression of fear scaling, experiment 2 took a within-subjects, optogenetic approach. Rats were NAcc-transducted with Halo or a control fluorophore, recovered, then acquired a scaled fear response to danger, uncertainty, and safety. Once scaling was established, rats received sessions in which the NAcc was illuminated during cue presentation or during the ITI. If the NAcc plays identical roles in the acquisition and expression of fear scaling, we would expect to observe a three-way interaction (group $\times$ illumination $\times$ cue) with only Halo rats showing impaired overall scaling during cue illumination sessions. If the NAcc plays a more selective role in the expression of rapid fear scaling, we would expect to observe a four-way interaction (group $\times$ interval $\times$ illumination $\times$ cue) with only Halo rats showing impaired rapid uncertaintysafety discrimination during cue illumination sessions.

\section{Histologic results}

Rats received bilateral NAcc transduction with halorhodopsin (Halo) or a control fluorophore (YFP) and bilateral optical ferrule implantation just above the NAcc. Representative transduction is shown (Fig. 3A). Each subject's total transduction area was drawn, made transparent, and stacked (Fig. 3B). Darker areas indicate regions of greater overlap and more consistent transduction. Transduction centered around and above the anterior commissure, the precise NAcc location.
Initial fear scaling

YFP and Halo rats acquired reliable fear scaling over the initial 10 sessions (Fig. $3 C$ ). Suppression ratios were low in preexposure and initially increased to all cues. As discrimination proceeded, the suppression ratio for each cue diverged: high to danger, intermediate to uncertainty, and low to safety. Demonstrating overall scaling, ANOVA [within factors: session (10) and 10-s cue (danger, uncertainty, and safety); between factor: group (YFP vs Halo)] revealed a main effect of cue $\left(F_{(2,46)}=36.21, p=\right.$ $3.58 \times 10^{-10}, \eta_{\mathrm{p}}^{2}=0.61$, op $\left.=1.00\right)$, session $\left(F_{(9,207)}=25.74\right.$, $\left.p=2.04 \times 10^{-29} \eta_{\mathrm{p}}^{2}=0.53, \mathrm{op}=1.00\right)$, and a cue $\times$ session interaction $\left(F_{(18,414)}=6.26, p=1.14 \times 10^{-13}, \eta_{\mathrm{p}}^{2}=0.21\right.$, op $=$ 1.00). ANOVA found no main effect or interaction with group (Fs $<3.42$, ps $>0.08)$. Thus, YFP and Halo entered the light illumination phase (Fig. $3 D, E$ ) showing equivalent fear scaling.

\section{Baseline nose poking}

YFP and Halo rats showed equivalent baseline nose poke rates throughout preexposure, discrimination, cable habituation, and light illumination (Fig. 4A). ANOVA for baseline nose poke rate [factors: session (20) and group (YFP vs Halo)] demonstrated a main effect of session $\left(F_{(19,437)}=12.60, p=4.19 \times 10^{-31}, \eta_{\mathrm{p}}^{2}=\right.$ 0.35 , op $=1.00$ ), but no main effect or interaction with group (Fs $<0.93$, ps $>0.55)$. Equivalent performance lessens the concern that between-group differences in cue suppression ratios result from differences in baseline nose poke rates. 

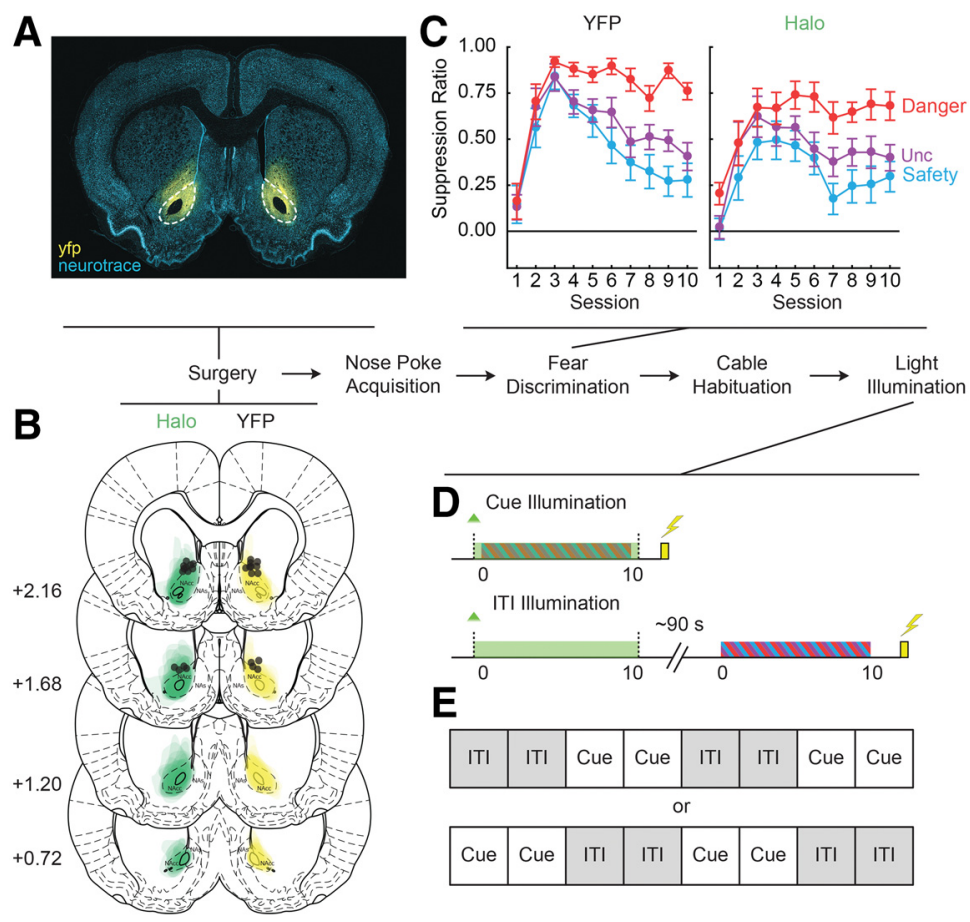

Figure 3. NAcc illumination experimental outline. $\boldsymbol{A}$, Representative NAcc transduction is shown with YFP expression (yellow fluorescent protein; yellow) and neurotrace (blue). Dotted lines approximate NAcc location. $\boldsymbol{B}$, The extent of viral transduction across four coronal planes is shown for Halo (green, left) and YFP rats (yellow, right), and the anterior distance from bregma (millimeters) indicated. Individual ferrule placement indicated in black circles. C, Mean \pm SEM suppression ratios for danger (red), uncertainty (purple), and safety (blue) are shown for YFP (left) and Halo rats (right) during the ten initial fear discrimination sessions. $\boldsymbol{D}$, In the final eight sessions, rats received NAcc light illumination during cue presentation (top) or during the ITI (bottom). Green indicates light illumination, yellow indicates shock delivery and candy-striped indicates cue presentation. $\boldsymbol{E}$, Cue and ITI illumination were given in alternating, two-session blocks. Block order was counterbalanced with roughly half of the subjects first receiving ITI illumination (top). NAs, nucleus accumbens shell.

\section{Overall fear scaling during light illumination}

When suppression ratios were calculated for the entire 10-s cue, YFP and Halo rats showed scaling of the fear response over the 10 sessions of cable habituation, cue illumination, and ITI illumination. ANOVA [between factor: group (YFP vs Halo); within factors: session (10) and cue (danger, uncertainty, and safety)] was separately performed for rats receiving ITI-cue illumination order (YFP, $n=5$; Halo, $n=5$; Fig. $4 B$ ) and cue-ITI illumination order (YFP, $n=7$; Halo, $n=8$; Fig. $4 C$ ). Each ANOVA returned a main effect of cue $\left(F s>29, p s<2 \times 10^{-7}\right)$, but neither returned a main effect of group, group $\times$ cue interaction or a group $\times$ cue $\times$ session interaction $(F s<2.5, p s>0.1)$. Complete ANOVA results provided in Table 1.

Next, we calculated difference scores for the two components of scaling: (danger - uncertainty) and (uncertainty - safety; Fig. 4D). ANOVA [between factors: group (YFP vs Halo) and order (ITI-cue vs cue-ITI); within factors: illumination (habituation/ ITI vs cue) and discrimination (danger - uncertainty vs uncertainty - safety)] found main effects of illumination $\left(F_{(1,21)}=8.90\right.$, $\left.p=0.007, \eta_{\mathrm{p}}^{2}=0.30, \mathrm{op}=0.81\right)$ and discrimination $\left(F_{(1,21)}=\right.$ $\left.14.29, p=0.001, \eta_{\mathrm{p}}^{2}=0.41, \mathrm{op}=0.95\right)$, as well as a group $\times$ illumination interaction $\left(F_{(1,21)}=4.75, p=0.041, \eta_{\mathrm{p}}^{2}=0.19\right.$, op $=$ $0.55)$. The interaction resulted from YFP rats showing poorer overall discrimination in cue illumination sessions compared with ITI illumination, whereas Halo rats showed equivalent discrimination in each session type. No main effect of group $\left(F_{(1,21)}\right.$ $=0.19, p=0.67, \eta_{\mathrm{p}}^{2}=0.009$, op $\left.=0.07\right)$ or any group interaction was detected $(F s<1.2, p s>0.3)$. These results reveal that NAcc activity is not necessary for the expression of fear scaling when suppression is measured for the duration of cues.

Rapid fear scaling during light illumination To examine rapid fear scaling, we divided the 10-s cue into five, 2-s intervals. Suppression ratios are shown for YFP (Fig. $5 A$ ) and Halo rats (Fig. 5B) for each cue/ interval during habituation/ITI and cue illumination sessions. We performed ANOVA with all factors [within factors: session-type (cable habituation, ITI illumination, and cue illumination), cue (danger, uncertainty, and safety), and interval (five, 2-s cue intervals); between factor: group (YFP vs Halo)]. The complete ANOVA output is reported in Table 2. Consistent with general scaling across groups, ANOVA revealed a main effect of cue $\left(F_{(2,46)}=89.04, p=1.53 \times 10^{-16}\right.$, $\left.\eta_{\mathrm{p}}^{2}=0.80, \mathrm{op}=1.00\right)$ as well as a cue $\times$ interval interaction $\left(F_{(8,184)}=6.14, p=5.16\right.$ $\left.\times 10^{-7}, \eta_{\mathrm{p}}^{2}=0.21, \mathrm{op}=1.00\right)$. Indicative of a selective role for the NAcc in rapid fear scaling, ANOVA revealed a significant four-way interaction [session-type $\times$ cue $\times$ interval $\times$ group $\left(F_{(16,368)}=1.80, p=0.029, \eta_{\mathrm{p}}^{2}=0.07\right.$, op $=0.95)]$, but not a significant three-way interaction [session-type $\times$ cue $\times$ group $\left(F_{(4,92)}=1.35, p=0.26, \quad \eta_{\mathrm{p}}^{2}=0.06\right.$, op $=$ $0.41)]$.

The four-way interaction indicates that YFP and Halo rats showed differing temporal scaling patterns across the different session types. To begin to clarify the differing patterns, we split YFP and Halo rats and performed identical ANOVAs [within factors: session-type (habituation, ITI illumination, and cue illumination), cue (danger, uncertainty, and safety), and interval (five, 2-s cue intervals)]. Indicative of reliable scaling, ANOVA for YFP rats found a main effect of cue $\left(F_{(2,22)}=47.71, p=1.0 \times 10^{-10}, \eta_{\mathrm{p}}^{2}=0.81\right.$, op $=$ $1.00)$ and a cue $\times$ interval interaction $\left(F_{(8,88)}=2.76, p=0.009\right.$, $\eta_{\mathrm{p}}^{2}=0.20$, op $\left.=0.92\right)$. Revealing no effect of illumination on the temporal pattern of fear scaling, the three-way interaction (session-type $\times$ cue $\times$ interval) was not significant $\left(F_{(16,176)}=0.59\right.$, $\left.p=0.89, \eta_{\mathrm{p}}^{2}=0.05, \mathrm{op}=0.39\right)$. ANOVA for Halo rats also found a main effect of cue $\left(F_{(2,24)}=41.39, p=1.66 \times 10^{-8}, \eta_{\mathrm{p}}^{2}=0.78\right.$, $\mathrm{op}=1.00)$ and a cue $\times$ interval interaction $\left(F_{(8,96)}=4.07\right.$, $p=3.36 \times 10^{-4}, \quad \eta_{\mathrm{p}}^{2}=0.25$, op $\left.=0.99\right)$. Only now, ANOVA revealed a significant three-way interaction (session-type $\times$ cue $\times$ interval; $F_{(16,192)}=1.92, p=0.021, \eta_{\mathrm{p}}^{2}=0.14$, op $\left.=0.95\right)$. NAcc illumination only disrupted the temporal scaling pattern for Halo rats.

As before, difference scores were calculated for the two components of scaling: (danger - uncertainty) and (uncertainty - safety). Separate scores were calculated for ITI illumination sessions and cue illumination sessions for the first 2-s interval (Fig. $5 C$ ) and the last 2-s interval (Fig. 5D). ANOVA [between factors: group (YFP vs Halo); within factors: interval (first $2 \mathrm{~s}$ and last 2 s), illumination (ITI vs cue) and discrimination (danger - uncertainty vs uncertainty - safety)] found a significant four-way interaction [interval $\times$ illumination $\times$ discrimination $\times$ group, $\left.\left(F_{(1,23)}=5.93, p=0.023, \eta_{\mathrm{p}}^{2}=0.21, \mathrm{op}=0.65\right)\right]$. ANOVA restricted to YFP rats found no significant three-way interaction 

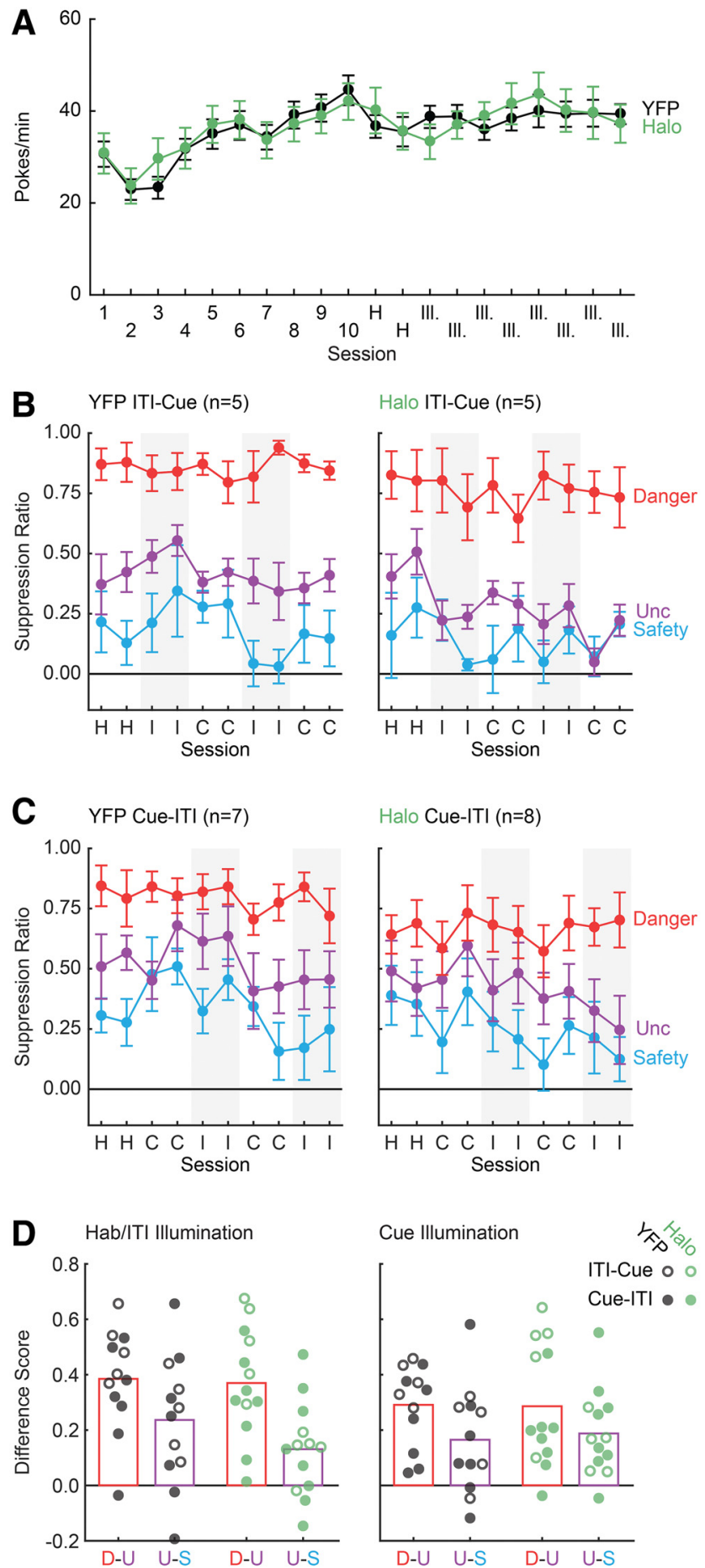

Figure 4. NAcc illumination and overall fear scaling. $A$, Mean \pm SEM nose poke rate is shown for YFP (black) and Halo rats (green) during the 10 preillumination (1-10), two cable habituation (H) and eight illumination (III.) sessions. $\boldsymbol{B}$, Mean \pm SEM suppression ratios over the entire 10-s cue are plotted for danger (red), uncertainty (purple), and safety (blue). Data are plotted for cable habituation (H), ITI illumination (I), and cue illumination (C) for YFP $(n=5)$ and Halo rats $(n=5)$ receiving ITI-cue illumination. ITI illumination sessions shaded. C, YFP $(n=7)$ and Halo rats $(n=8)$ receiving cue-ITI illumination plotted as in $\boldsymbol{A}$. $\boldsymbol{D}$, Difference scores for danger versus uncertainty ( $D-U$, red bar) and uncertainty versus safety (U-S, purple bar) are shown for YFP (black) and Halo rats (green) during cable habituation/ ITI illumination (left) and cue illumination (right). ITI-cue rats indicated by open circles, cueITI rats by closed circles.
Table 1. Complete ANOVA results for NAcc illumination and overall fear scaling

\begin{tabular}{|c|c|c|c|c|c|c|c|c|}
\hline \multirow[b]{2}{*}{ Term } & \multicolumn{4}{|l|}{ ITI-Cue } & \multicolumn{4}{|c|}{ Cue-ITI } \\
\hline & $F$ & $p$ & $\eta_{\mathrm{p}}^{2}$ & op & $F$ & $p$ & $\eta_{p}^{2}$ & op \\
\hline Cue & 103.37 & $7.09 \times 10^{-10}$ & 0.93 & 1.00 & 29.23 & $2.23 \times 10^{-7}$ & 0.69 & 1.00 \\
\hline Cue $\times$ group & 0.55 & 0.59 & 0.06 & 0.13 & 0.15 & 0.86 & 0.01 & 0.07 \\
\hline Session & 1.55 & 0.15 & 0.16 & 0.68 & 3.46 & 0.001 & 0.21 & 0.98 \\
\hline Session $\times$ group & 2.04 & 0.047 & 0.2 & 0.82 & 0.77 & 0.65 & 0.06 & 0.37 \\
\hline Cue $\times$ session & 1.21 & 0.26 & 0.13 & 0.79 & 1.07 & 0.39 & 0.08 & 0.74 \\
\hline $\begin{array}{l}\text { Cue } \times \text { session } \times \\
\text { group }\end{array}$ & 1.18 & 0.29 & 0.13 & 0.78 & 1.47 & 0.10 & 0.10 & 0.90 \\
\hline Group & 2.51 & 0.15 & 0.24 & 0.29 & 1.19 & 0.30 & 0.08 & 0.17 \\
\hline
\end{tabular}

ANOVA was performed for suppression ratio over the 10-s cue with factors of group, session, and cue for (top) rats receiving the ITI-Cue illumination order and (bottom) rats receiving the Cue-ITI illumination order. $F$ statistic, $p$ value, $\eta_{\mathrm{p}}^{2}$ and observed power (op) are reported for every main effect and interaction.

[interval $\times$ illumination $\times$ discrimination, $\left(F_{(1,11)}=1.68, p=\right.$ $0.22, \eta_{\mathrm{p}}^{2}=0.13$, op $\left.\left.=0.22\right)\right]$ while Halo rats just missed statistical significance [interval $\times$ illumination $\times$ discrimination, $\left(F_{(1,12)}=\right.$ 4.47, $p=0.056, \eta_{\mathrm{p}}^{2}=0.27$, op $\left.\left.=0.49\right)\right]$.

These results suggest that cue illumination, but not ITI, impaired rapid uncertainty-safety discrimination in Halo rats. By contrast, YFP rats showed equivalent, rapid uncertainty-safety discrimination during cue and ITI illumination sessions. To directly examine this, we calculated an illumination difference score during the first and last 2-s cue intervals: [(uncertaintysafety discrimination during Cue illumination) - (uncertaintysafety discrimination during ITI illumination)] (Fig. 5E). The illumination difference score capitalizes on our within-subject design: each rat was tested under ITI and cue illumination conditions. The illumination difference score simplifies the complex interaction by summarizing the differential effects of cue and ITI illumination in a single value. Values around zero indicate equivalent uncertainty-safety discrimination during cue and ITI illumination sessions. Negative values indicate worse uncertainty-safety discrimination during cue illumination sessions. Two individuals (one YFP and one Halo) had first-interval illumination difference scores \pm 2 SDs beyond the group mean. The data for these individuals are shown (Fig. $5 E$, open circles), but were not included in $t$ test analyses.

Halo rats showed worse uncertainty-safety discrimination during cue illumination sessions compared with ITI illumination sessions during the first 2-s cue interval (Fig. $5 E$, left). This was supported by a significant, negative shift of illumination difference scores away from zero (one-sample $t$ test, $t_{(11)}=-3.65$, $p=0.004$ ). YFP rats showed equivalent uncertainty-safety discrimination during cue and ITI illumination sessions $\left(t_{(10)}=\right.$ 1.22, $p=0.25)$. Further, YFP and Halo illumination difference scores differed from one another (independent samples $t$ test, $\left.t_{(21)}=3.22, p=0.004\right)$. Impaired uncertainty-safety discrimination in Halo rats receiving cue illumination was restricted to the first 2-s cue interval. Identical analysis of the last 2-s cue interval found that illumination difference scores did not differ from zero for Halo (one-sample $t$ test, $t_{(11)}=0.27, p=0.80$ ) or YFP rats (one-sample $t$ test, $t_{(10)}=-0.41, p=0.69$; Fig. $5 E$, right), which did not differ from one another (independent samples $t$ test, $t_{(21)}$ $=0.48, p=0.64)$. Altogether, the results reveal that optogenetic inhibition of NAcc cue activity impairs the expression of rapid uncertainty-safety discrimination.

Of course, it is possible that NAcc optogenetic inhibition simply suppressed rewarded nose poking. In this case, impaired rapid fear scaling would be the by-product of a general reduction in poking. To rule out this possibility, we analyzed nose poke suppression during light illumination in ITI sessions (Fig. 5F). 

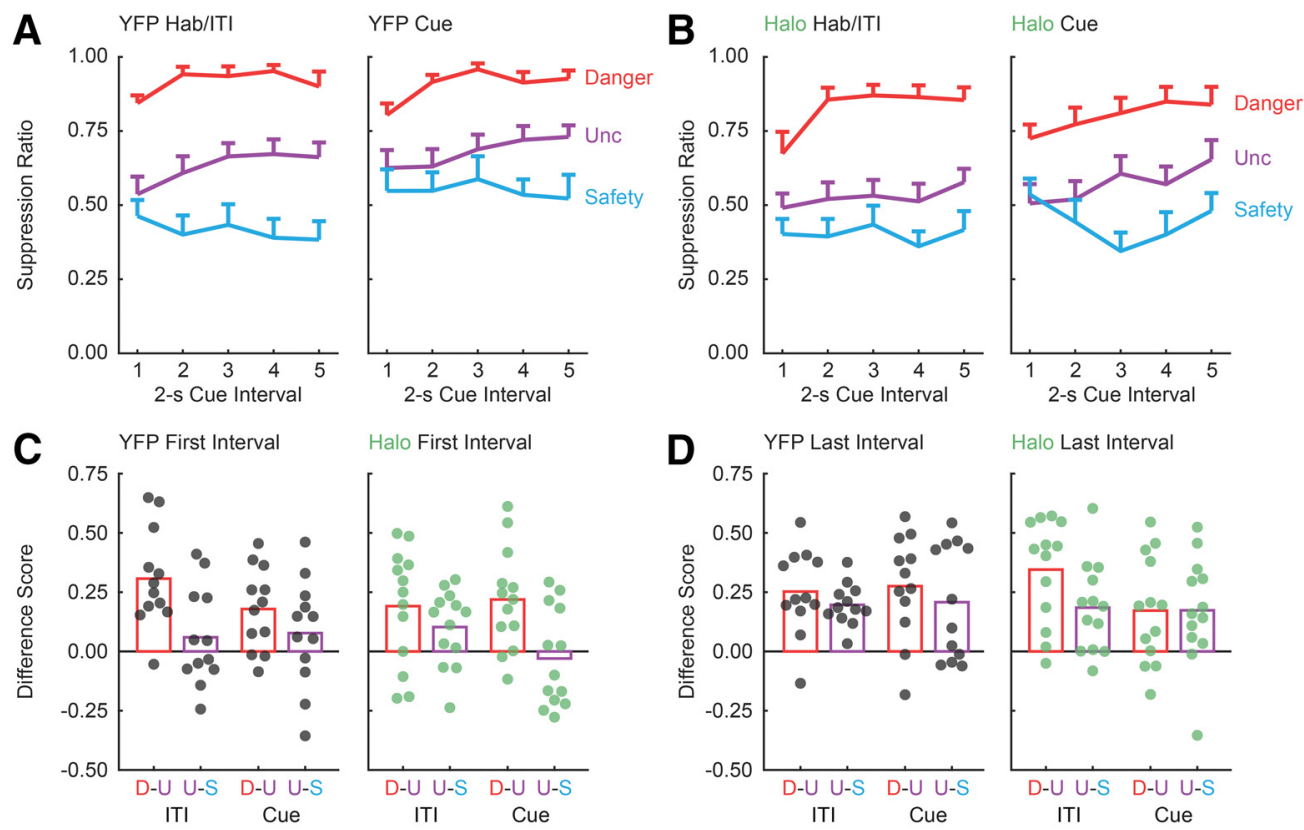

Halo Last Interval
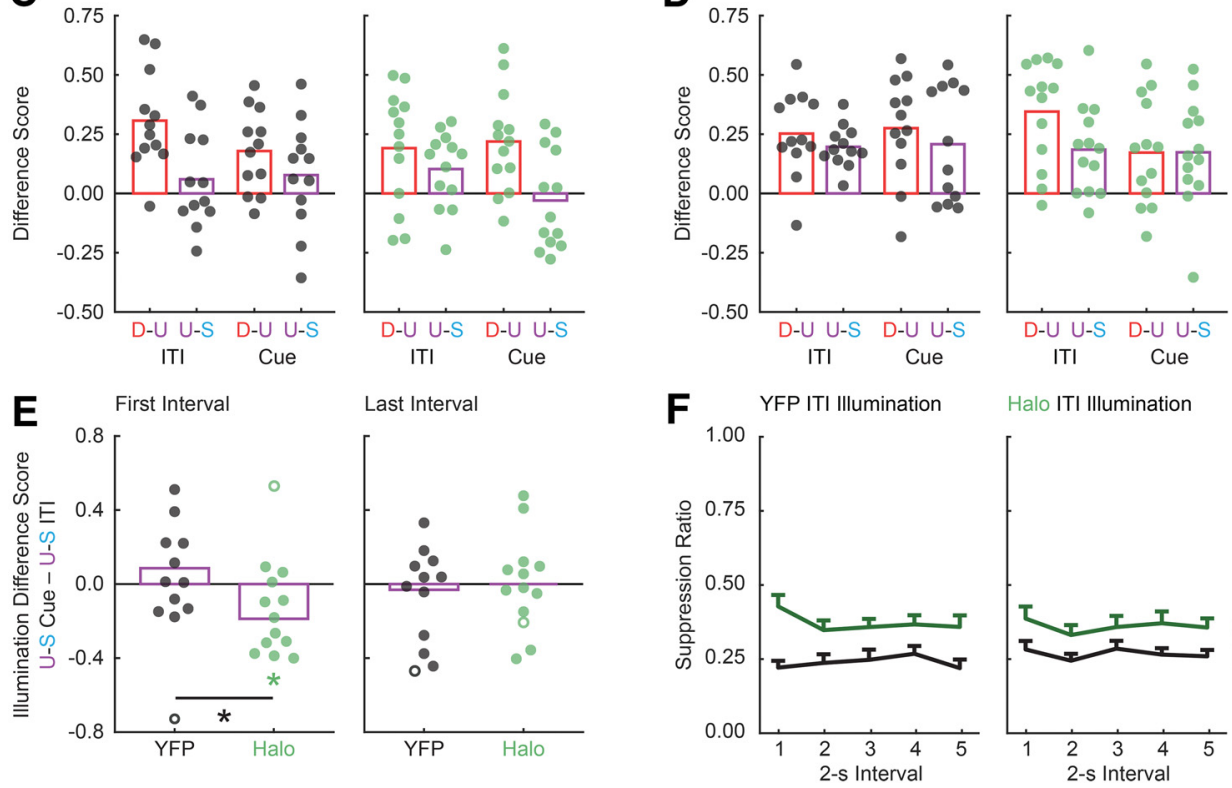

Halo ITI Illumination

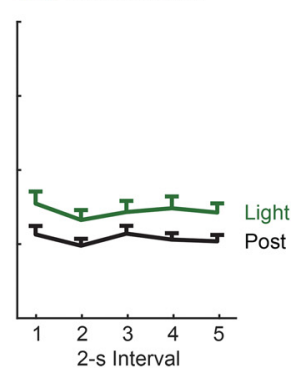

Figure 5. NAcc illumination and rapid fear scaling. Mean \pm SEM suppression ratio is plotted for the five, 2-s cue intervals for danger (red), uncertainty (purple), and safety (blue), for $(\boldsymbol{A})$ YFP and $(\boldsymbol{B})$ Halo rats during cable habituation/ITI illumination (left), and cue illumination (right). $\boldsymbol{C}$, Mean difference score for danger versus uncertainty (D-U, red bar) and uncertainty versus safety (U-S, purple bar) for the first 2-s cue interval is shown for YFP (left, black circles) and Halo rats (right, black circles). Data points show individual difference scores. $\boldsymbol{D}$, Difference score data for the last 2 -s cue interval shown as in $\boldsymbol{C}$. $\boldsymbol{E}$, Difference scores were separately calculated for ITI and cue illumination, then an illumination difference was calculated (cue difference score - ITI difference score). Mean and individual illumination difference scores are plotted for the first 2-s cue interval (left) and last 2-s cue interval (right), for YFP (black) and Halo rats (green). Open circles are outliers; ${ }^{*}$ (green) one-sample $t$ test compared with zero, $p=0.0038$; ${ }^{*}$ (black) independent samples $t$ test, $p=0.0041$. $\boldsymbol{F}$, Mean \pm SEM suppression ratios are plotted for the five, 2-s intervals during ITI illumination (dark green) and for the five, 2-s intervals during the postillumination period (black; YFP, left; Halo, right).

Table 2. Complete ANOVA results for NAcc illumination and rapid fear scaling

\begin{tabular}{lrlcl}
\hline Term & \multicolumn{1}{l}{$l$} & $p$ & $\eta_{\mathrm{p}}^{2}$ & op \\
\hline Group & 1.94 & 0.18 & 0.08 & 0.27 \\
Session-type & 10.64 & $1.59 \times 10^{-4}$ & 0.32 & 0.99 \\
Session-type $\times$ group & 2.22 & 0.12 & 0.09 & 0.43 \\
Cue & 89.04 & $1.53 \times 10^{-16}$ & 0.80 & 1.00 \\
Cue $\times$ group & 0.57 & 0.57 & 0.02 & 0.14 \\
Interval & 6.00 & $2.46 \times 10^{-4}$ & 0.21 & 0.98 \\
Interval $\times$ group & 1.37 & 0.25 & 0.06 & 0.41 \\
Session-type $\times$ cue & 4.21 & 0.004 & 0.16 & 0.91 \\
Session-type $\times$ cue $\times$ group & 1.35 & 0.26 & 0.06 & 0.41 \\
Session-type $\times$ interval & 0.71 & 0.68 & 0.03 & 0.32 \\
Session-type $\times$ interval $\times$ group & 1.46 & 0.17 & 0.06 & 0.65 \\
Cue $\times$ interval & 6.14 & $5.16 \times 10^{-7}$ & 0.21 & 1.00 \\
Cue $\times$ interval $\times$ group & 0.64 & 0.74 & 0.03 & 0.29 \\
Session-type $\times$ cue $\times$ interval & 0.78 & 0.71 & 0.03 & 0.54 \\
Session-type $\times$ cue $\times$ interval $\times$ group & 1.80 & 0.029 & 0.07 & 0.95 \\
\hline Ald & & &
\end{tabular}

ANOVA was performed for suppression ratio over the five, 2-s cue intervals with factors of group, sessiontype, cue, and interval. $F$ statistic, $p$ value, $\eta_{p}^{2}$, and observed power (op) are reported for every main effect and interaction.
No cues were present during this period, allowing us to determine the effect of light illumination alone to suppress nose poking. The middle $10 \mathrm{~s}$ of the 11-s light illumination was divided into five, 2-s intervals, exactly as was done for the cue illumination analyses. For comparison, we also sampled 10 s of nose poking $30 \mathrm{~s}$ following illumination offset. This postillumination period served as a control to which light illumination could be compared. ANOVA [within factors: period (light and post) and interval (five, 2-s intervals); between factor: group (YFP vs Halo)] revealed main effects of period $\left(F_{(1,23)}=34.53, p=5 \times\right.$ $\left.10^{-6}, \eta_{\mathrm{p}}^{2}=0.60, \mathrm{op}=1.00\right)$ and interval $\left(F_{(4,92)}=2.49, p=0.049\right.$, $\eta_{\mathrm{p}}^{2}=0.10$, op $\left.=0.69\right)$. Critically, ANOVA found no main effect or interaction with group $(F s<1.10, p s>0.31)$. Thus, while suppression ratios were higher during light illumination, this did not differ between YFP and Halo rats and was therefore not due to inhibition of NAcc activity.

\section{Discussion}

We set out to examine a role for the NAcc in fear scaling. Neurotoxic lesions revealed a general role for the NAcc in the 
acquisition of fear scaling, as well as a specific role in acquiring rapid uncertainty-safety discrimination. Optogenetic inhibition revealed a specific role for NAcc cue activity in the expression of rapid, uncertainty-safety discrimination. The results reveal that the NAcc is an essential component of a neural circuit permitting fear to scale to degree of threat.

Before discussing our results more broadly, we must consider several limitations of our experimental design and results. First, our experiments only used male rats. Several studies have reported sex differences in danger-safety discrimination (Day et al., 2016; Foilb et al., 2018; Greiner et al., 2019). We find only modest sex differences in our discrimination procedure (Walker et al., 2018, 2019), suggesting similar neural circuits may be used across sexes. Of course, females and males may achieve similar performance through differing neural mechanisms. Another important consideration is that our dependent measure of fear is derived from the rate of rewarded nose poking. Conditioned suppression is a strength because it provides an objective measure of fear on multiple time scales (Estes and Skinner, 1941; Bouton and Bolles, 1980). It is a potential weakness because the NAcc plays a well-established role in reward-seeking. Disrupting NAcc function can attenuate reward-related behavior in many settings (Corbit et al., 2001; Hall et al., 2001; Ito et al., 2004; Blaiss and Janak, 2009; Ambroggi et al., 2011; McDannald et al., 2011, 2013), although this finding is not universal (Ramirez and Savage, 2007; Corbit and Balleine, 2011). In our first experiment, NAcc lesions slowed the increase in baseline nose poking over discrimination sessions and also impaired fear scaling. However, the deficits in reward-seeking and fear scaling did not align. The fear scaling deficit was apparent across all sessions, while the nose poking deficit only emerged in later sessions.

Our second experiment better demonstrated independent roles for the NAcc in rapid fear scaling and reward-seeking. Light illumination during the cue period impaired rapid uncertainty-safety discrimination in NAcc-Halo rats but not NAccYFP rats. By contrast, light illumination during the ITI produced equivalent and modest reductions in nose poking for both groups. Optogenetic inhibition of the NAcc was insufficient to reduce rewarded nose poking. The failure of NAcc inhibition to suppress nose poking may seem odd. Mice will readily perform actions that channelrhodopsin-excite NAcc D1 and D2 cell types (Cole et al., 2018), and rats will perform actions that channelrhodopsin-excite NAcc glutamatergic inputs (Stuber et al., 2011; Britt et al., 2012). However, these studies demonstrate that NAcc activity is sufficient, but not necessary, to support reward-seeking. Prominent theories posit that reward-seeking initially depends on medial striatal structures, such as the NAcc. With further training, lateral striatal regions (e.g., dorsolateral striatum) control reward-seeking (Gerdeman et al., 2003; Belin and Everitt, 2008; Corbit et al., 2012; Burton et al., 2015). In our second experiment, rats had extensive experience with nose poking when the NAcc was optogenetically inhibited. By this time, reward-seeking may have no longer been under NAcc control, yet the NAcc continued to contribute to rapid fear scaling. In another intriguing reward tie-in, dopamine bursts, "blips" onto D1R-NAcc neurons promote cue-reward generalization while dopamine pauses, "dips" onto D2R-NAcc neurons promote cuereward discrimination (Iino et al., 2020). Receptor-and cell-typespecific dopamine shaping of NAcc threat responding would be an appealing future research direction and is perhaps likely to occur (Badrinarayan et al., 2012; Jo et al., 2018).

A role for the NAcc in fear would be expected based on immediate early gene studies. Cues and context associated with foot shock reliably upregulate NAcc c-fos and zif268 (Beck and Fibiger, 1995; Campeau et al., 1997; Thomas et al., 2002). Despite these clear findings, pinpointing the role of the NAcc in fear has been challenging. Initial work by Parkinson and colleagues found that NAcc lesions impaired cued fear, but enhanced contextual fear (Parkinson et al., 1999). Taking a similar experimental approach, Levita and colleagues found that NAcc lesions had no impact on the acquisition or expression of cued fear, but impaired retention of contextual fear (Levita et al., 2002). Contemporary work by Haralambous and Westbrook (1999) found that inhibiting accumbens activity (core + shell) specifically impaired the acquisition, but not expression of contextual fear, and had no effect on cued fear. Even considering slightly different methodologies, it is difficult to reconcile these disparate results.

These are not the only conflicts in the literature. Schwienbacher and colleagues found that blocking NAcc activity with tetrodotoxin abolished the acquisition, and impaired the expression, of fear-potentiated startle (Schwienbacher et al., 2004). The very next year, Josselyn and colleagues used a variety of methods to manipulate the NAcc during fear-potentiated startle: lesion, agonizing dopamine, and blocking glutamate. NAcc manipulation had no effect on any aspect of fear-potentiated startle (Josselyn et al., 2005). Since these initial studies, the NAcc has been implicated in a variety of fear-related processes. For example, the NAcc can modulate salience: the ability of cues to enter into associations with foot shock (Iordanova et al., 2006a,b; Iordanova, 2009). Human imaging studies have observed NAcc correlates of prediction error, a theoretical signal that strengthens or weakens cue-shock associations (Seymour et al., 2004; Delgado et al., 2008; Schiller et al., 2008; Li et al., 2011), consistent with a role for the NAcc in predictive learning ( $\mathrm{Li}$ and McNally, 2015).

What can we make of the mixed NAcc fear literature? Although dissatisfying, one answer is that the NAcc must play multiple roles. Genetically and anatomically defined NAcc neuron types may be linked to specific fear processes. Future work dissecting the NAcc in this way, as has been done in reward settings (Kupchik et al., 2015; Francis and Lobo, 2017; Tejeda et al., 2017) is likely to be fruitful. A more fulfilling answer might be that standard cued and contextual fear conditioning procedures do not isolate essential NAcc functions. The NAcc may not be necessary to demonstrate fear to certain threat or to withhold fear to certain safety. For example, the NAcc is not necessary to behaviorally discriminate contexts/cues associated with certain shock and certain safety (Antoniadis and McDonald, 2006; McDannald and Galarce, 2011; Piantadosi, 2017).

We propose that a necessary role for the NAcc in fear emerges when subjects are confronted with threats on a continuum from safety to danger. The NAcc is a core component of a neural circuit permitting the level of the fear response to scale to degree of threat. During acquisition, the NAcc is generally necessary for fear scaling over the duration of an encounter, in our case for the entirety of cue presentation. At the same time, the NAcc is specifically necessary for one component of fear scaling: rapid discrimination of uncertain threat and safety. Once a scaled fear response is acquired, the general role for the NAcc diminishes. However, the NAcc continues to play a specific role in rapidly discriminating uncertain threat and safety. Of course, we are not claiming that fear scaling is the sole function of the NAcc in fear, but rather a function.

Environmental threats are not absolute, but exist on a continuum from safety to danger. Using a behavioral procedure that 
attempts to capture this continuum, we find the NAcc is essential to scale fear to degree of threat. Our results clarify at least one role for the NAcc in fear, yet much more work remains. NAcc structure and function is altered in anxiety and stress disorders (Cha et al., 2014; Felmingham et al., 2014; Manning et al., 2015; Morey et al., 2017). Disrupted threat-safety discrimination may be conceptualized as maladaptive fear scaling. Recent work shows that NAcc resting state functional connectivity is highly conserved across mice, macaques and humans (Balsters et al., 2020). Preclinical research detailing NAcc threat function, and mapping a more complete neural circuit for fear scaling, is likely to inform strategies to promote adaptive fear in anxiety and stress disorders.

\section{References}

Ambroggi F, Ghazizadeh A, Nicola SM, Fields HL (2011) Roles of nucleus accumbens core and shell in incentive-cue responding and behavioral inhibition. J Neurosci 31:6820-6830.

Antoniadis EA, McDonald RJ (2006) Fornix, medial prefrontal cortex, nucleus accumbens, and mediodorsal thalamic nucleus: roles in a fear-based context discrimination task. Neurobiol Learn Mem 85:71-85.

Badrinarayan A, Wescott SA, Vander Weele CM, Saunders BT, Couturier BE, Maren S, Aragona BJ (2012) Aversive stimuli differentially modulate real-time dopamine transmission dynamics within the nucleus accumbens core and shell. J Neurosci 32:15779-15790.

Balsters JH, Zerbi V, Sallet J, Wenderoth N, Mars RB (2020) Primate homologs of mouse cortico-striatal circuits. Elife 9:e53680.

Beck CH, Fibiger HC (1995) Conditioned fear-induced changes in behavior and in the expression of the immediate early gene c-fos: with and without diazepam pretreatment. J Neurosci 15:709-720.

Belin D, Everitt BJ (2008) Cocaine seeking habits depend upon dopamine-dependent serial connectivity linking the ventral with the dorsal striatum. Neuron 57:432-441.

Berg BA, Schoenbaum G, McDannald MA (2014) The dorsal raphe nucleus is integral to negative prediction errors in Pavlovian fear. Eur J Neurosci 40:3096-3101.

Blaiss CA, Janak PH (2009) The nucleus accumbens core and shell are critical for the expression, but not the consolidation, of Pavlovian conditioned approach. Behav Brain Res 200:22-32.

Bouton ME, Bolles RC (1980) Conditioned fear assessed by freezing and by the suppression of three different baselines. Anim Learn Behav 8:429434.

Britt JP, Benaliouad F, McDevitt RA, Stuber GD, Wise RA, Bonci A (2012) Synaptic and behavioral profile of multiple glutamatergic inputs to the nucleus accumbens. Neuron 76:790-803.

Burton AC, Nakamura K, Roesch MR (2015) From ventral-medial to dorsallateral striatum: neural correlates of reward-guided decision-making. Neurobiol Learn Mem 117:51-59.

Campeau S, Falls WA, Cullinan WE, Helmreich DL, Davis M, Watson SJ (1997) Elicitation and reduction of fear: behavioural and neuroendocrine indices and brain induction of the immediate-early gene $\mathrm{c}$-fos. Neuroscience 78:1087-1104.

Cha J, Carlson JM, DeDora DJ, Greenberg T, Proudfit GH, Mujica-Parodi LR (2014) Hyper-reactive human ventral tegmental area and aberrant mesocorticolimbic connectivity in overgeneralization of fear in generalized anxiety disorder. J Neurosci 34:5855-5860.

Cole SL, Robinson MJF, Berridge KC (2018) Optogenetic self-stimulation in the nucleus accumbens: $d 1$ reward versus D2 ambivalence. PLoS One 13: e0207694.

Corbit LH, Balleine BW (2011) The general and outcome-specific forms of pavlovian-instrumental transfer are differentially mediated by the nucleus accumbens core and shell. J Neurosci 31:11786-11794.

Corbit LH, Muir JL, Balleine BW (2001) The role of the nucleus accumbens in instrumental conditioning: evidence of a functional dissociation between accumbens core and shell. J Neurosci 21:3251-3260.

Corbit LH, Nie H, Janak PH (2012) Habitual alcohol seeking: time course and the contribution of subregions of the dorsal striatum. Biol Psychiatry 72:389-395.
Correia SS, McGrath AG, Lee A, Graybiel AM, Goosens KA (2016) Amygdala-ventral striatum circuit activation decreases long-term fear. Elife 5:e12669.

Cromwell HC, Schultz W (2003) Effects of expectations for different reward magnitudes on neuronal activity in primate striatum. J Neurophysiol 89:2823-2838.

Day HLL, Reed MM, Stevenson CW (2016) Sex differences in discriminating between cues predicting threat and safety. Neurobiol Learn Mem 133:196-203.

Delgado MR, Li J, Schiller D, Phelps EA (2008) The role of the striatum in aversive learning and aversive prediction errors. Philos Trans R Soc Lond B Biol Sci 363:3787-3800.

DiLeo A, Wright KM, McDannald MA (2016) Sub-second fear discrimination in rats: adult impairment in adolescent heavy alcohol drinkers. Learn Mem 23:618-622.

Duits P, Cath DC, Lissek S, Hox JJ, Hamm AO, Engelhard IM, van den Hout MA, Baas JM (2015) Updated meta-analysis of classical fear conditioning in the anxiety disorders. Depress Anxiety 32:239-253.

Estes KW, Skinner BF (1941) Some quantitative properties of anxiety. J Exp Psychol 29:390-400

Fadok JP, Darvas M, Dickerson TMK, Palmiter RD (2010) Long-term memory for Pavlovian fear conditioning requires dopamine in the nucleus accumbens and basolateral amygdala. PLoS One 5:e12751.

Felmingham KL, Falconer EM, Williams L, Kemp AH, Allen A, Peduto A, Bryant RA (2014) Reduced amygdala and ventral striatal activity to happy faces in PTSD is associated with emotional numbing. PLoS One 9: e103653.

Foilb AR, Bals J, Sarlitto MC, Christianson JP (2018) Sex differences in fear discrimination do not manifest as differences in conditioned inhibition. Learn Mem 25:49-53.

Francis TC, Lobo MK (2017) Emerging role for nucleus accumbens medium spiny neuron subtypes in depression. Biol Psychiatry 81:645-653.

Gerdeman GL, Partridge JG, Lupica CR, Lovinger DM (2003) It could be habit forming: drugs of abuse and striatal synaptic plasticity. Trends Neurosci 26:184-192.

Goosens KA, Maren S (2001) Contextual and auditory fear conditioning are mediated by the lateral, basal, and central amygdaloid nuclei in rats. Learn Mem 8:148-155.

Greiner EM, Müller I, Norris MR, Ng KH, Sangha S (2019) Sex differences in fear regulation and reward-seeking behaviors in a fear safety-reward discrimination task. Behav Brain Res 368:111903.

Hall J, Parkinson JA, Connor TM, Dickinson A, Everitt BJ (2001) Involvement of the central nucleus of the amygdala and nucleus accumbens core in mediating Pavlovian influences on instrumental behaviour. Eur J Neurosci 13:1984-1992.

Haralambous T, Westbrook RF (1999) An infusion of bupivacaine into the nucleus accumbens disrupts the acquisition but not the expression of contextual fear conditioning. Behav Neurosci 113:925-940.

Iino Y, Sawada T, Yamaguchi K, Tajiri M, Ishii S, Kasai H, Yagishita S (2020) Dopamine D2 receptors in discrimination learning and spine enlargement. Nature 579:555-560

Iordanova MD (2009) Dopaminergic modulation of appetitive and aversive predictive learning. Rev Neurosci 20:383-404.

Iordanova MD, McNally GP, Westbrook RF (2006a) Opioid receptors in the nucleus accumbens regulate attentional learning in the blocking paradigm. J Neurosci 26:4036-4045.

Iordanova MD, Westbrook RF, Killcross AS (2006b) Dopamine activity in the nucleus accumbens modulates blocking in fear conditioning. Eur J Neurosci 24:3265-3270

Ito R, Robbins TW, Everitt BJ (2004) Differential control over cocaine-seeking behavior by nucleus accumbens core and shell. Nat Neurosci 7:389397.

Jo YS, Heymann G, Zweifel LS (2018) Dopamine neurons reflect the uncertainty in fear generalization. Neuron 100:916-925.e3.

Josselyn SA, Falls WA, Gewirtz JC, Pistell P, Davis M (2005) The nucleus accumbens is not critically involved in mediating the effects of a safety signal on behavior. Neuropsychopharmacology 30:17-26.

Jovanovic T, Norrholm SD, Blanding NQ, Davis M, Duncan E, Bradley B, Ressler KJ (2010) Impaired fear inhibition is a biomarker of PTSD but not depression. Depress Anxiety 27:244-251.

Jovanovic T, Kazama A, Bachevalier J, Davis M (2012) Impaired safety signal learning may be a biomarker of PTSD. Neuropharmacology 62:695-704. 
Kita H, Kitai ST (1990) Amygdaloid projections to the frontal-cortex and the striatum in the rat. J Comp Neurol 298:40-49.

Koo JW, Han JS, Kim JJ (2004) Selective neurotoxic lesions of basolateral and central nuclei of the amygdala produce differential effects on fear conditioning. J Neurosci 24:7654-7662.

Kupchik YM, Brown RM, Heinsbroek JA, Lobo MK, Schwartz DJ, Kalivas PW (2015) Coding the direct/indirect pathways by D1 and D2 receptors is not valid for accumbens projections. Nat Neurosci 18:1230-1232.

Levita L, Dalley JW, Robbins TW (2002) Disruption of Pavlovian contextual conditioning by excitotoxic lesions of the nucleus accumbens core. Behav Neurosci 116:539-552.

Li J, Schiller D, Schoenbaum G, Phelps EA, Daw ND (2011) Differential roles of human striatum and amygdala in associative learning. Nat Neurosci $14: 1250-1252$.

Li SSY, McNally GP (2015) A role of nucleus accumbens dopamine receptors in the nucleus accumbens core, but not shell, in fear prediction error. Behav Neurosci 129:450-456.

Lissek S, Kaczkurkin AN, Rabin S, Geraci M, Pine DS, Grillon C (2014) Generalized anxiety disorder is associated with overgeneralization of classically conditioned fear. Biol Psychiatry 75:909-915.

Manning J, Reynolds G, Saygin ZM, Hofmann SG, Pollack M, Gabrieli JD, Whitfield-Gabrieli S (2015) Altered resting-state functional connectivity of the frontal-striatal reward system in social anxiety disorder. PLoS One 10:e 0125286.

McDannald MA, Galarce EM (2011) Measuring Pavlovian fear with conditioned freezing and conditioned suppression reveals different roles for the basolateral amygdala. Brain Res 1374:82-89.

McDannald MA, Lucantonio F, Burke KA, Niv Y, Schoenbaum G (2011) Ventral striatum and orbitofrontal cortex are both required for modelbased, but not model-free, reinforcement learning. J Neurosci 31:27002705.

McDannald MA, Setlow B, Holland PC (2013) Effects of ventral striatal lesions on first- and second-order appetitive conditioning. Eur J Neurosci 38:2589-2599.

McGinty VB, Lardeux S, Taha SA, Kim JJ, Nicola SM (2013) Invigoration of reward seeking by cue and proximity encoding in the nucleus accumbens. Neuron 78:910-922.

Morey RA, Davis SL, Garrett ME, Haswell CC; Mid-Atlantic MIRECC Workgroup, Marx CE, Beckham JC, McCarthy G, Hauser MA, AshleyKoch AE (2017) Genome-wide association study of subcortical brain volume in PTSD cases and trauma-exposed controls. Transl Psychiatry $7: 1265$

Oleson EB, Gentry RN, Chioma VC, Cheer JF (2012) Subsecond dopamine release in the nucleus accumbens predicts conditioned punishment and its successful avoidance. J Neurosci 32:14804-14808.

Ottenheimer D, Richard JM, Janak PH (2018) Ventral pallidum encodes relative reward value earlier and more robustly than nucleus accumbens. Nat Commun 9:4350.

Parkinson JA, Olmstead MC, Burns LH, Robbins TW, Everitt BJ (1999) Dissociation in effects of lesions of the nucleus accumbens core and shell on appetitive pavlovian approach behavior and the potentiation of conditioned reinforcement and locomotor activity by D-amphetamine. J Neurosci 19:2401-2411.

Petrovich GD, Risold PY, Swanson LW (1996) Organization of projections from the basomedial nucleus of the amygdala: a PHAL study in the rat. J Comp Neurol 374:387-420.
Piantadosi PT (2017) Contributions of nucleus accumbens circuitry to aspects of aversively-motivated behaviors. $\mathrm{PhD}$ thesis, University of British Columbia.

Quirk GJ, Repa C, LeDoux JE (1995) Fear conditioning enhances short-latency auditory responses of lateral amygdala neurons: parallel recordings in the freely behaving rat. Neuron 15:1029-1039.

Ramirez DR, Savage LM (2007) Differential involvement of the basolateral amygdala, orbitofrontal cortex, and nucleus accumbens core in the acquisition and use of reward expectancies. Behav Neurosci 121:896-906.

Ray MH, Hanlon E, McDannald MA (2018) Lateral orbitofrontal cortex partitions mechanisms for fear regulation and alcohol consumption. PLoS One 13:e0198043.

Rescorla RA (1968) Probability of shock in the presence and absence of CS in fear conditioning. J Comp Physiol Psychol 66:1-5.

Saddoris MP, Carelli RM (2014) Cocaine self-administration abolishes associative neural encoding in the nucleus accumbens necessary for higherorder learning. Biol Psychiatry 75:156-164.

Schiller D, Levy I, Niv Y, LeDoux JE, Phelps EA (2008) From fear to safety and back: reversal of fear in the human brain. J Neurosci 28:1151711525 .

Schwienbacher I, Fendt M, Richardson R, Schnitzler HU (2004) Temporary inactivation of the nucleus accumbens disrupts acquisition and expression of fear-potentiated startle in rats. Brain Res 1027:87-93.

Setlow B, Schoenbaum G, Gallagher M (2003) Neural encoding in ventral striatum during olfactory discrimination learning. Neuron 38:625-636.

Seymour B, O’Doherty JP, Dayan P, Koltzenburg M, Jones AK, Dolan RJ, Friston KJ, Frackowiak RS (2004) Temporal difference models describe higher-order learning in humans. Nature 429:664-667.

Stuber GD, Sparta DR, Stamatakis AM, van Leeuwen WA, Hardjoprajitno JE, Cho S, Tye KM, Kempadoo KA, Zhang F, Deisseroth K, Bonci A (2011) Excitatory transmission from the amygdala to nucleus accumbens facilitates reward seeking. Nature 475:377-380.

Sugam JA, Saddoris MP, Carelli RM (2014) Nucleus accumbens neurons track behavioral preferences and reward outcomes during risky decision making. Biol Psychiatry 75:807-816.

Tejeda HA, Wu J, Kornspun AR, Pignatelli M, Kashtelyan V, Krashes MJ, Lowell BB, Carlezon WA, Bonci A (2017) Pathway-and cell-specific kappa-opioid receptor modulation of excitation-inhibition balance differentially gates D1 and D2 accumbens neuron activity. Neuron 93:147163.

Thomas KL, Hall J, Everitt BJ (2002) Cellular imaging with zif268 expression in the rat nucleus accumbens and frontal cortex further dissociates the neural pathways activated following the retrieval of contextual and cued fear memory. Eur J Neurosci 16:1789-1796.

Walker RA, Andreansky C, Ray MH, McDannald MA (2018) Early adolescent adversity inflates threat estimation in females and promotes alcohol use initiation in both sexes. Behav Neurosci 132:171-182.

Walker RA, Wright KM, Jhou TC, McDannald MA (2019) The ventrolateral periaqueductal grey updates fear via positive prediction error. Eur J Neurosci 51:866-880.

Wright CI, Groenewegen HJ (1996) Patterns of overlap and segregation between insular cortical, intermediodorsal thalamic and basal amygdaloid afferents in the nucleus accumbens of the rat. Neuroscience 73:359-373.

Wright KM, DiLeo A, McDannald MA (2015) Early adversity disrupts the adult use of aversive prediction errors to reduce fear in uncertainty. Front Behav Neurosci 9:227. 